\title{
Patrones de distribución temporal de algunas variables físicas y químicas en el embalse Paso Bonito, Cienfuegos, Cuba
}

\author{
Carmen Betancourt ${ }^{1, *}$, Roberto Suárez ${ }^{2}$ y Liliana Toledo ${ }^{1}$ \\ ${ }^{1}$ Centro de Estudios Ambientales de Cienfuegos.Calle 17, esq. Ave 46 s/n, Reparto Reina, Cienfuegos 55100, \\ Cuba. \\ ${ }^{2}$ Universidad de Cienfuegos. Carretera a Rodas km 3. C.P. 59430 Cienfuegos, Cuba. \\ * Autor responsable de la correspondencia: carmen@gestion.ceac.cu
}

Recibido: 21/5/08

Aceptado: 8/10/08

\begin{abstract}
Temporal distribution patterns of some physical and chemical variables in the Paso Bonito reservoir, Cienfuegos, Cuba A study is presented on the $\mathrm{pH}$, redox potential (Eh), dissolved oxygen (DO), and temperature (T) in the Paso Bonito reservoir, used as a source of drinking water supply for the Cienfuegos City. This reservoir has its basin located mainly in a mountain area, affected by the organic, physical, and residue pollution from an old pyrite mine. From September 2006 through November 2007 samples were collected at meter intervals from the surface to bottom near water withdrawal site. The reservoir was polymictic during wet summers, and monomictic during dry summers. During thermal stratification it had a clinograde oxygen distribution pattern, a pH between 7.0 and 8.1 and a negative hypolimnetic Eh during thermal stratification. We derived an empiric model relating Eh, DO and T. The correlations between the studied indicators were also determined. These results will facilitate the interpretation of highly complex phenomena such as the release of nutrients, metals, and organic matter from the sediments.
\end{abstract}

Key words: Temperature, $\mathrm{pH}$, Redox potential, dissolved oxygen, thermal stratification.

\section{RESUMEN}

Patrones de distribución temporal de algunas variables físicas y químicas en el embalse de Paso Bonito (Cienfuegos, Cuba)

Se presenta un estudio de pH, potencial redox (Eh), oxígeno disuelto (OD) y temperatura (T) en el embalse Paso Bonito, utilizado para el abastecimiento de agua potable a la Ciudad de Cienfuegos. Este embalse tiene su cuenca de alimentación ubicada fundamentalmente en una zona de montaña, afectada por la contaminación orgánica, física y de residuos de una antigua mina de pirita. El muestreo fue realizado en la columna de agua del punto de toma del embalse, desde la superficie hasta el fondo, metro a metro, desde Septiembre de 2006 hasta Noviembre de 2007. El embalse estudiado presenta una distribución térmica de tipo polimíctico, en veranos de abundantes precipitaciones y monomíctico en veranos secos; con un patrón de distribución de oxígeno de tipo clinógrado; un pH entre 7.0 y 8.1, y un Eh negativo en el hipolimnion en períodos de estratificación térmica. Se ha realizado un modelo empírico que relaciona el Eh con el OD y la T. Se determinaron además las correlaciones entre los indicadores estudiados. Estos resultados facilitarán la interpretación de fenómenos altamente complejos como la liberación de nutrientes, metales y materia orgánica desde los sedimentos.

Palabras clave: Temperatura, pH, potencial redox, oxígeno disuelto, estratificación térmica. 


\section{INTRODUCCIÓN}

El conocimiento de las características físicoquímicas del agua de un embalse constituye una herramienta importante para gestionar adecuadamente este recurso. Dentro de los indicadores a tener en cuenta, la temperatura, el potencial redox (Eh), el oxígeno disuelto (OD) y el $\mathrm{pH}$, resultan de gran interés porque se incluyen dentro de los factores que regulan el fenómeno altamente complejo de liberación de nutrientes, metales y materia orgánica desde los sedimentos (Boström et al., 1988; Aminot \& Andrieux, 1996; Appan \& Ting, 1996; Harris, 1999).

La temperatura es un factor abiótico que controla procesos vitales para los organismos, así como también afecta las propiedades químicas y físicas de otros factores abióticos en un ecosistema. A su vez, incrementos de este indicador pueden alargar los períodos de estratificación, potenciando el efecto de los contaminantes aportados por la cuenca. (Armengol y García, 1997).

El oxígeno disuelto es importante en los procesos de fotosíntesis, oxidación-reducción, solubilidad de minerales y la descomposición de materia orgánica. La anoxia hipolimnética puede traer consecuencias negativas sobre la calidad del agua, como es el caso de la liberación de nutrientes (amonio y ortofosfato) y metales desde el fondo (Boström et al., 1988; Kilham \& Kilham, 1990; Ahlgren et al., 1994; Kassim, 1997, McGinnis \& Little, 2002; Beutel, 2006).

El Eh regula el comportamiento de muchos compuestos químicos presentes en cuerpos de agua naturales. La solubilidad de metales como hierro y manganeso y el estado de oxidación de nutrientes como el nitrógeno, carbono y azufre dependen del Eh y el pH del medio.

Tilman et al., (1982) definieron al pH como la variable principal de la química de los sistemas acuáticos. El mismo informa sobre aspectos tan interesantes como son las características litológicas de la cuenca de drenaje, los usos del suelo, o la actividad biológica que se desarrolla en un cuerpo de agua. Los cambios en el $\mathrm{pH}$ pueden ser usados para estimar el metabolismo de los sistemas acuáticos (Geider \& Osborne, 1992) y la dinámica de los nutrientes (Harper, 1992).
El embalse Paso Bonito, fuente de abastecimiento de la Ciudad de Cienfuegos, se está afectando por contaminantes derivados de malas prácticas de manejo (Betancourt y Toledo, 2007), los cuales pueden incidir en el comportamiento de variables tales como OD, Eh y pH. A su vez, incrementos de la $\mathrm{T}$ pueden alargar los períodos de estratificación potenciando el efecto de los contaminantes aportados por la cuenca.

El presente trabajo tiene como objetivo conocer el patrón de distribución de la tempertaura, $\mathrm{pH}$, oxígeno disuelto y Eh en la columna de agua del embalse, para poder interpretar posteriormente los resultados obtenidos en investigaciones relacionadas con el comportamiento de variables como hierro, manganeso, ciclo del fósforo, ciclo del nitrógeno y distribución de fitoplancton.

\section{MATERIAL Y MÉTODOS}

\section{Área de estudio}

El Embalse Paso Bonito está ubicado al sureste de la provincia de Cienfuegos, región centro-sur de Cuba (Fig. 1). Fue construido en 1975 como la principal fuente de abastecimiento de la Ciudad de Cienfuegos y parte de la Ciudad de Santa Clara. En el mismo se acumulan las aguas del río Hanabanilla y las procedentes de una hidroeléctrica ubicada en el embalse Hanabanilla a través de un túnel.

Su cuenca yace sobre mármoles grises azulosos, esquistos carbonatados y graníticos, esquistos cuarzos moscovíticos con capas aisladas de mármol y rocas magmáticas, y está ubicada en una zona de montaña y premontaña, resultando vulnerable en lo que a erosión y transporte de contaminantes se refiere.

En la cuenca se ubican asentamientos humanos, despulpadora de café, vaquerías y áreas de cultivos (fundamentalmente café), entre otras actividades antrópicas. Algunas características de la cuenca y del embalse, se informan en la Tabla 1.

\section{Muestreo}

Se realizaron nueve muestreos a partir de Septiembre de 2006 hasta Noviembre de 2007, in- 


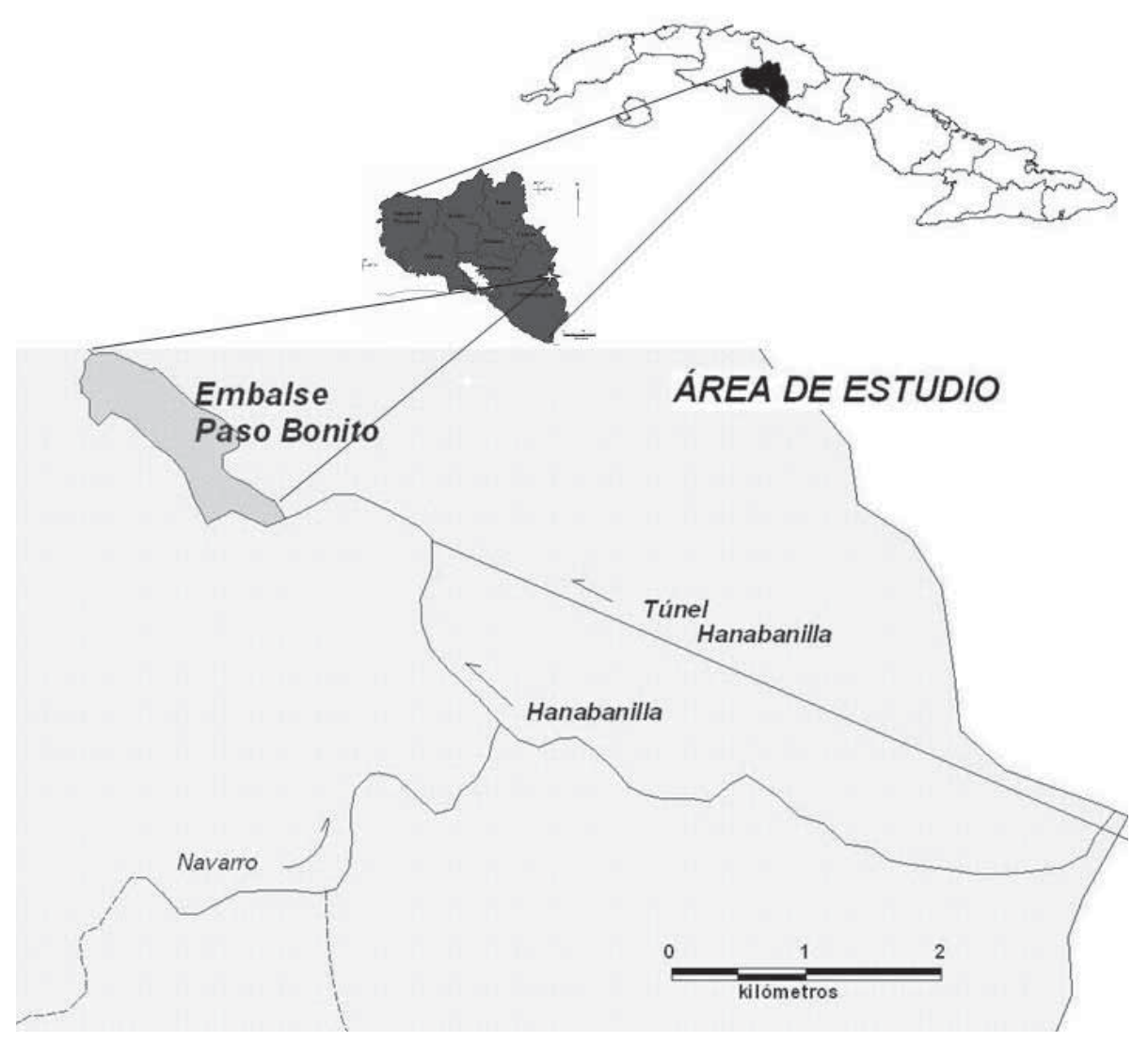

Figura 1. Área de estudio. Study site.

cluyendo los meses de principio y final de los supuestos períodos de mezcla y estratificación térmica del embalse (Septiembre, Octubre, Noviembre, Diciembre, de 2006 y Febrero, Mayo, Julio y Octubre de 2007 respectivamente).

Las muestras se tomaron primeramente en la superficie y a continuación metro a metro hasta el fondo en un perfil ubicado en el punto de toma del embalse $(n=99)$ y se determinaron las siguientes variables: profundidad $(\mathrm{m})$, temperatura del agua $\left({ }^{\circ} \mathrm{C}\right), \mathrm{pH}$ (unidades), oxígeno disuelto $(\mathrm{mg} / \mathrm{l})$, porcentaje de saturación de OD y Eh $(\mathrm{mV})$.

Para la toma de muestras se utilizó una botella Niskin de cinco litros de capacidad. Las determinaciones de $\mathrm{pH}$ y Eh se realizaron según el manual del equipo HANNA HI 9025-C, la temperatura fue medida in situ y el OD se determinó por el método Winkler.

\section{Análisis de datos}

Los porcentajes de saturación de oxígeno disuelto se calcularon a partir de la solubilidad del oxígeno en función de la temperatura y la

Tabla 1. Características de la zona de estudio. Study site characteristics.

Área de la cuenca $\left(\mathrm{km}^{2}\right)^{*} \quad 65$

Altitud media de la cuenca $(\mathrm{m})^{*} \quad 187$

Altitud del embalse (m) $\quad 86.3$

Volumen $\left(\mathrm{hm}^{3}\right) \quad 8$

Area $\left(\mathrm{km}^{2}\right) \quad 1.25$

Escurrimiento medio anual $\left(\mathrm{hm}^{3}\right)$

Longitud del río principal $(\mathrm{km}) \quad 16.5$

* Datos tomados del archivo de la Delegación de Recursos Hidráulicos en Cienfuegos 
Betancourt et al.

Tabla 2. Porcentaje de saturación oxígeno. Oxygen saturation percentage.

\begin{tabular}{lclc}
\hline & máximo $\%$ de $\mathrm{O}_{2}$ y profundidad a la que se produce & Mínimo $\%$ de $\mathrm{O}_{2}$ \\
\hline Septiembre & 104 & $(1 \mathrm{~m})$ & 0 \\
Octubre de 2007 & 68 & $(1 \mathrm{~m})$ & 0 \\
Noviembre de 2006 & 87 & $(1 \mathrm{~m})$ & 52 \\
Diciembre & 92 & $($ Superficie $)$ & 61 \\
Febrero & 104 & $(1 \mathrm{~m})$ & 36 \\
Mayo & 92 & $($ Superficie $)$ & 33 \\
Julio & 113 & $(1 \mathrm{~m})$ & 0 \\
\hline
\end{tabular}

salinidad, según APHA (1992), (Tabla 2). Se excluyeron los valores registrados en Octubre 2006 y Noviembre 2007 por resultar superiores respecto a estos mismos meses de los años 2007 y 2006 respectivamente.

Para describir cualitativamente la calidad del agua en función del porcentaje de saturación del oxígeno disuelto se tuvo en cuenta el criterio de Lynch y Poole (1979) (citado por Fuentes y Massol-Dayá, 2002) (Tabla 3).

Para el trabajo estadístico se comprobó la normalidad de los datos, las diferencias significativas entre los indicadores evaluados durante el estudio se corroboraron a partir de una comparación de media metro a metro, verificando las hipótesis planteadas a través de la prueba $t$ de Student.

Las diferencias estadísticamente significativas entre el $\mathrm{pH}$ de Febrero y los meses restantes fueron establecidas mediante pruebas de hipótesis sobre la media en cada una de las profundidades; de igual forma se procedió con el $\mathrm{pH}$ de $\mathrm{Ma}-$ yo y el Eh de Octubre de 2007. Se excluyeron las mediciones de $\mathrm{pH}$ de Mayo para la prueba de Febrero y viceversa.

Tabla 3. Calidad del agua en función del porcentaje de saturación de Oxígeno. Water quality according to the oxygen saturation percentage.

\begin{tabular}{lc}
\hline Calidad & $\begin{array}{c}\text { Porcentaje de saturación de OD (a la T } \\
\text { y salinidad prevaleciente en el ambiente) }\end{array}$ \\
\hline Buena & $>90$ \\
Regular & $89-75$ \\
Dudosa & $74-50$ \\
Contaminada & $<50$ \\
\hline
\end{tabular}

* Criterio de Lynch y Poole (1979), citado por Fuentes y Massol-Dayá, 2002.
El análisis de la influencia de la profundidad y los procesos de estratificación térmica y mezcla sobre el OD, se hizo aplicando un análisis de varianza con ambos factores. Debido a la presencia de interacción entre ambos factores, se realizó un análisis de varianza univariado para establecer las diferencias entre las distintas combinaciones.

Para estudiar la relación entre el Eh y el resto de las variables en estudio, se utilizaron inicialmente diagramas de dispersión y análisis de correlaciones mediante el coeficiente de correlación de Pearson. Posteriormente se utilizó la regresión lineal múltiple de la variable Eh sobre las variables independientes, hasta obtener un modelo con las variables que mejor explican la relación.

\section{RESULTADOS}

\section{Temperatura}

El proceso de estratificación térmica alcanzó su mayor estabilidad en el mes de Octubre de 2006 (Fig. 2), caracterizado por un metalimnion que se ubica entre los 4 y $6 \mathrm{~m}$ de profundidad, con un gradiente de $\mathrm{T}$ de $2.2^{\circ} \mathrm{C}$.

Con la llegada del invierno en Noviembre, comenzó el proceso de mezcla del embalse que se extendió hasta Mayo. En Julio se observaron diferencias de temperatura superiores a $1{ }^{\circ} \mathrm{C}$ entre 1 y $2 \mathrm{~m}$ de profundidad.

En Octubre de 2007, se observó un gradiente térmico entre el primer y segundo metro de profundidad y entre los 9 y $10 \mathrm{~m}$ (fondo del embalse), con un proceso de mezcla entre los 2 y $9 \mathrm{~m}$. Este comportamiento es anómalo si se considera que Octubre coincide con el fin del verano en 


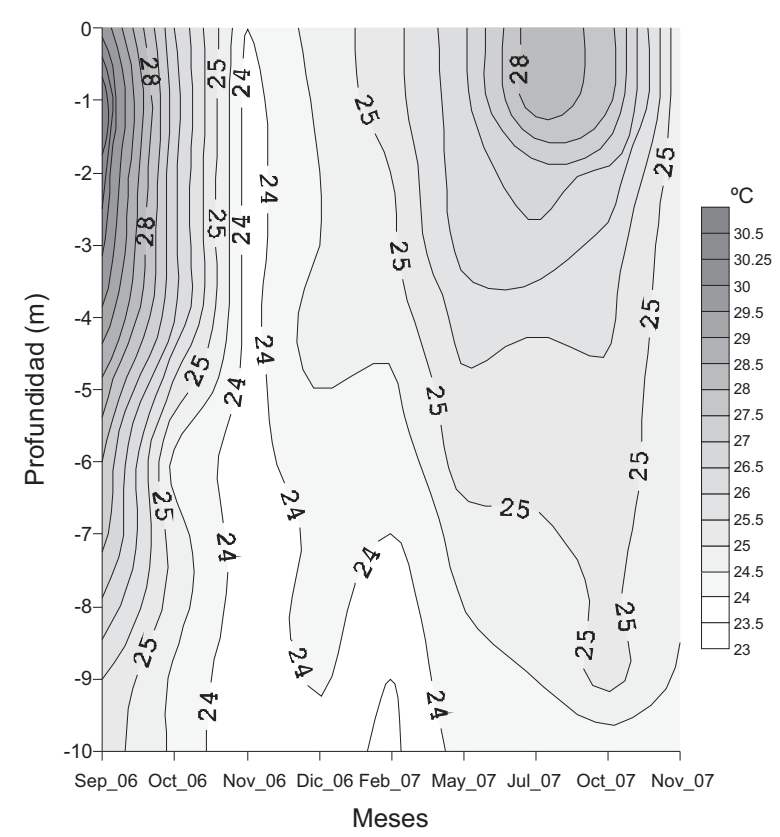

Figura 2. Ciclo térmico del embalse de Paso Bonito. Thermal cycle of the Paso Bonito Reservoir.

Cuba y durante el año anterior se observó la mayor estratificación del embalse en este mes.

\section{Oxígeno Disuelto}

Las concentraciones de oxígeno disuelto durante la campaña oscilaron en el intervalo de 0 a $8.66 \mathrm{mg} / \mathrm{l}$ (Fig. 3). De forma general los valores más bajos en la columna de agua se registraron en la zona próxima al fondo y durante los meses de Octubre, mientras que los más altos se encontraron a un metro de profundidad.

Los mayores gradientes de concentración (oxiclina) por meses de muestreo, se observaron en las capas más superficiales (1-6 m) durante los meses de Julio, Septiembre y Octubre de 2006. En Febrero, Mayo y Noviembre los gradientes se registraron en el fondo, mientras que en Diciembre, las disminuciones son menos acentuadas. Oxiclina y termoclina coinciden en los meses de Julio, Septiembre y Octubre de 2006.

En el fondo del embalse el agua resultó contaminada según los criterios de clasificación expuestos en la Tabla 3, con porcentajes de saturación menores que 50 para todos los meses, excepto para Noviembre (2006 y 2007) y Diciembre que re-

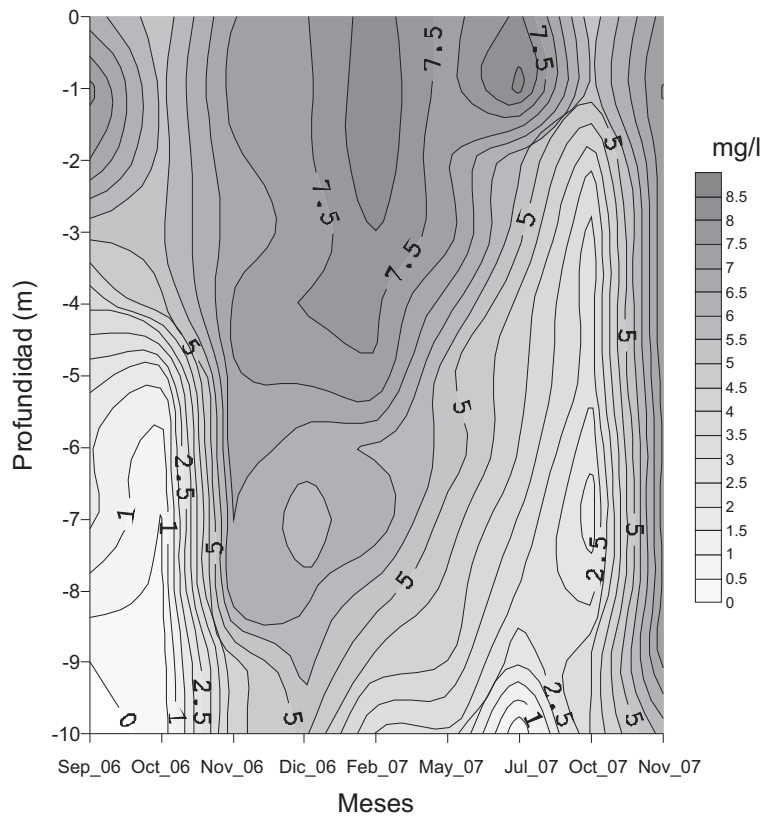

Figura 3. Evolución temporal y vertical en la concentración de oxígeno. Temporal and vertical evolution of the oxygen conentration.

sultó dudosa, (74-50\%). En el mes de Febrero, a pesar de que el embalse estaba circulando, también se registró una baja saturación en el fondo $(35.5 \%)$ que indica contaminación del agua.

En Octubre de 2007 la calidad del agua en toda la columna es dudosa o contaminada, lo que se deduce de los porcentajes de saturación de oxígeno relativamente bajas, entre $67.5 \%$ y $21.1 \%$ y un patrón de distribución vertical normal, donde los valores disminuyen desde la superficie hasta los $7 \mathrm{~m}$ y aumentan en $0.66 \mathrm{mg} / \mathrm{l} \mathrm{a}$ los $8 \mathrm{~m}$, con una posterior disminución gradual de dicho indicador hacia el fondo (Fig. 3).

En la prueba Anova para establecer el efecto de interacción de las distintas combinaciones de niveles de profundidad (superficie, medio y fondo) con los procesos de estratificación térmica y mezcla sobre las concentraciones medias de oxígeno, se obtuvieron los siguientes resultados:

1. No se observó diferencia significativa entre la superficie, medio y fondo, durante el proceso de mezcla del embalse.

2. Mientras ocurría la estratificación térmica del embalse se encontraron diferencias sig- 
nificativas al comparar superficie con medio $(p<0.031)$ y fondo $(p=0.000)$, pero no entre la profundidad media y fondo.

3. Al comparar ambos procesos (mezcla y estratificación térmica en conjunto), se encontró diferencia significativa $(p<0.031)$ entre el medio (estratificación térmica) y superficie (mezcla); fondo (estratificación térmica) y superficie (mezcla) ( $p=0.000)$; y fondo (estratificación térmica) y medio (mezcla) $(p=0.000)$.

Estas consideraciones ponen de manifiesto que el déficit de OD durante la estratificación térmica se verifica desde las profundidades medias del embalse donde se ubica el punto de toma.

\section{Potencial Redox}

El $85.7 \%$ de las mediciones durante el estudio se enmarcaron entre los 90 y $200 \mathrm{mV}$ (Fig. 4). El mayor valor $(200 \mathrm{mV})$ se registró en el mes de Noviembre de 2006 cuando el embalse estaba circulando, mientras que las cifras más bajas corresponden con el período de estratificación térmica (Julio, Septiembre y Octubre de 2006), llegando a alcanzar valores negativos en la zonas próximas al fondo del embalse $(-125.5 \mathrm{mV}) \mathrm{du}-$ rante el mes de Octubre de 2006.

En Julio, cuando se inicia el proceso de estratificación térmica del embalse, se observa un gradiente del indicador (redoxclina) entre los 9 y $10 \mathrm{~m}$, mientras que el gradiente de oxígeno se observa entre 1 y $2 \mathrm{~m}$. A medida que avanza el proceso de estratificación térmica, la oxiclina comienza a descender y la redoxclina a ascender de tal manera que hacia finales de la estratificación térmica (Octubre de 2006) coinciden la oxiclina, termoclina y redoxclina. Esta coincidencia en la columna de agua no resulta común (García-Gil y Camacho, 2001).

Al comparar el Eh del mes de Octubre de 2007 (valores bajos y bastante homogéneos en la columna de agua) con el resto de los meses, se obtuvieron diferencias estadísticamente significativas $(p \leq 0.032)$, corroborando que el Eh es inferior al resto de los meses estudiados desde la superfi-

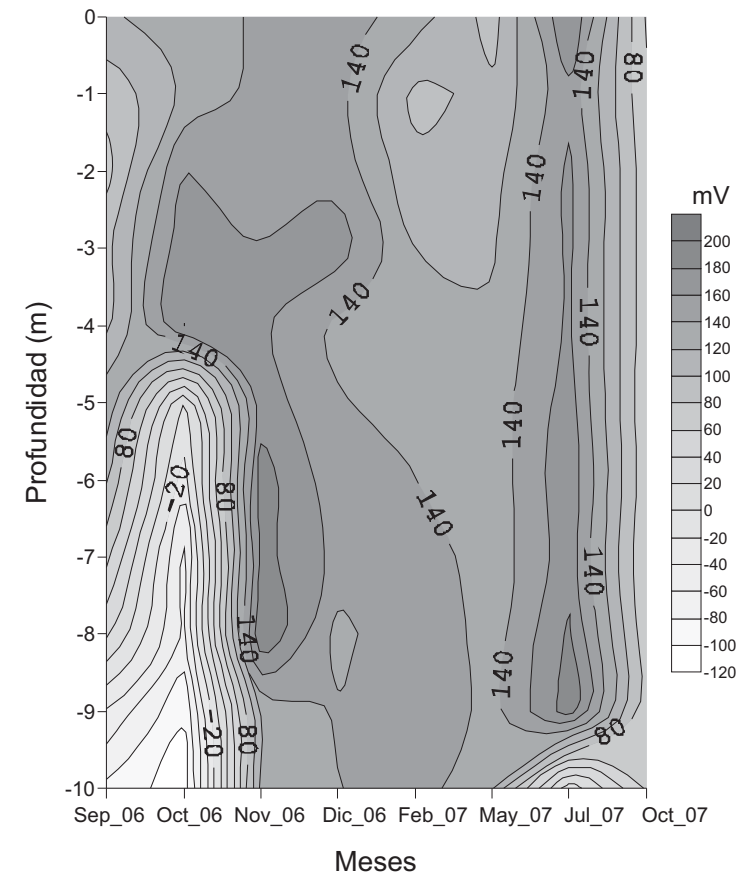

Figura 4. Evolución temporal y vertical del potencial redox. Temporal and vertical evolution of Redox potential.

cie hasta los $6 \mathrm{~m}$ de profundidad. En Diciembre y Mayo también se observó homogeneidad en toda la columna de agua, pero con valores más altos.

Adicionalmente, en este propio mes, el Eh registró una disminución gradual hasta los $8 \mathrm{~m}$, aumentó ligeramente en los $9 \mathrm{~m}$ y alcanzó valores más bajos a los $10 \mathrm{~m}$. Esta anomalía se puede observar en la figura 4.

\section{Modelo empírico para el Eh}

Considerando que el Eh es una variable dependiente del $\mathrm{pH}$, la temperatura y la concentración de oxígeno, se realizaron gráficos de dispersión para explorar la naturaleza de la relación entre ellos.

En la regresión del Eh sobre el resto de las variables en estudio, fue excluida la variable $\mathrm{pH}$ con el empleo del método paso a paso. Sin embargo, existen autores (Wagman et al., 1982 y Bachmanm et al., 2001) que han encontrado ecuaciones dependientes del $\mathrm{pH}$. Por su parte, Chen et al., (1983), (citado por Wallace, 2007) encontraron ecuaciones que incluyen además del pH, el oxígeno.

En este estudio se obtuvieron tres modelos; uno que incluyó todo el período evaluado, otro 


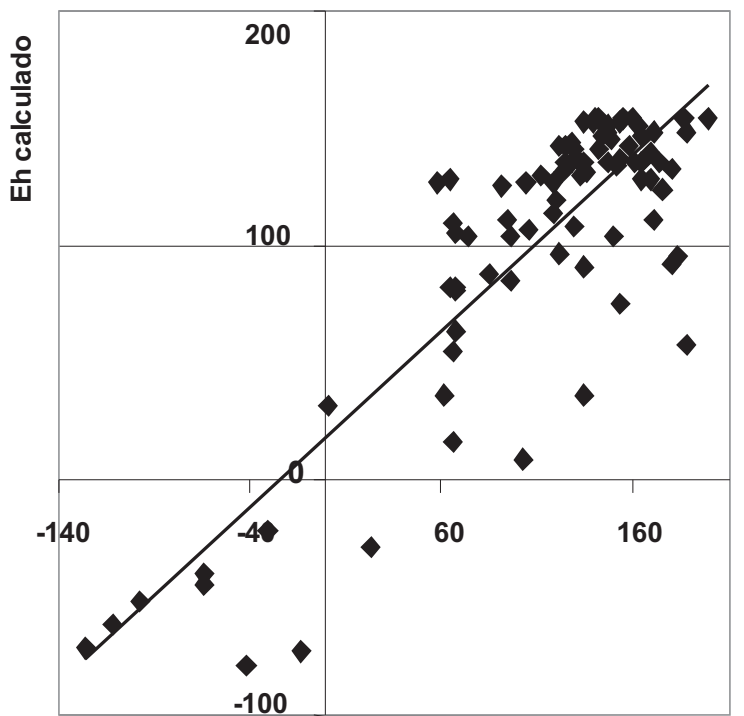

Eh observado

Figura 5. Comparación de los valores de Eh observados y calculados mediante el modelo (mezcla y estratificación térmica). Comparisson of the Eh values observed and calculated by the model (mixing and thermal stratification).

durante el proceso de mezcla del embalse, y otro para la estratificación, todos dependientes de la concentración de oxígeno y de la temperatura.

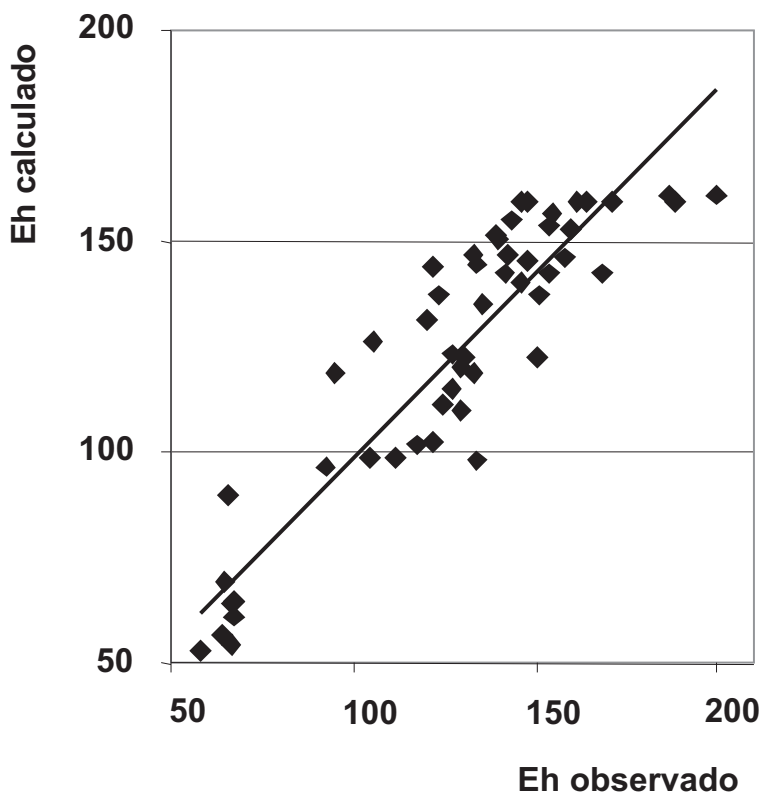

Figura 6. Comparación de los valores de Eh observados y calculados mediante el modelo (mezcla). Comparisson of the Eh values observed and calculated by the mode (mixing).
El modelo que se obtuvo para el período de estratificación térmica mostró una baja correlación, por lo que fue excluido de los resultados.

Modelo considerando todo el período evaluado.

$E h=91.8+76.154 O D-6.492(O D)^{2}-6.687 T$.

$\left(r^{2}=0.798\right)$

Modelo para el período de mezcla.

$E h=597.64+38.335 O D-2.69(O D)^{2}-24.146 T$.

$\left(r^{2}=0.808\right)$

La relación entre el Eh observado y el obtenido mediante los modelos durante los procesos de estratificación térmica y mezcla se muestran en las figuras 5 y 6 respectivamente.

\section{pH}

Los valores de $\mathrm{pH}$ en todas las mediciones realizadas se enmarcan en el intervalo de 7.11 (Septiembre) a 8.50 (Mayo), es decir entre ligeramente alcalino y alcalino respectivamente (Fig. 7).

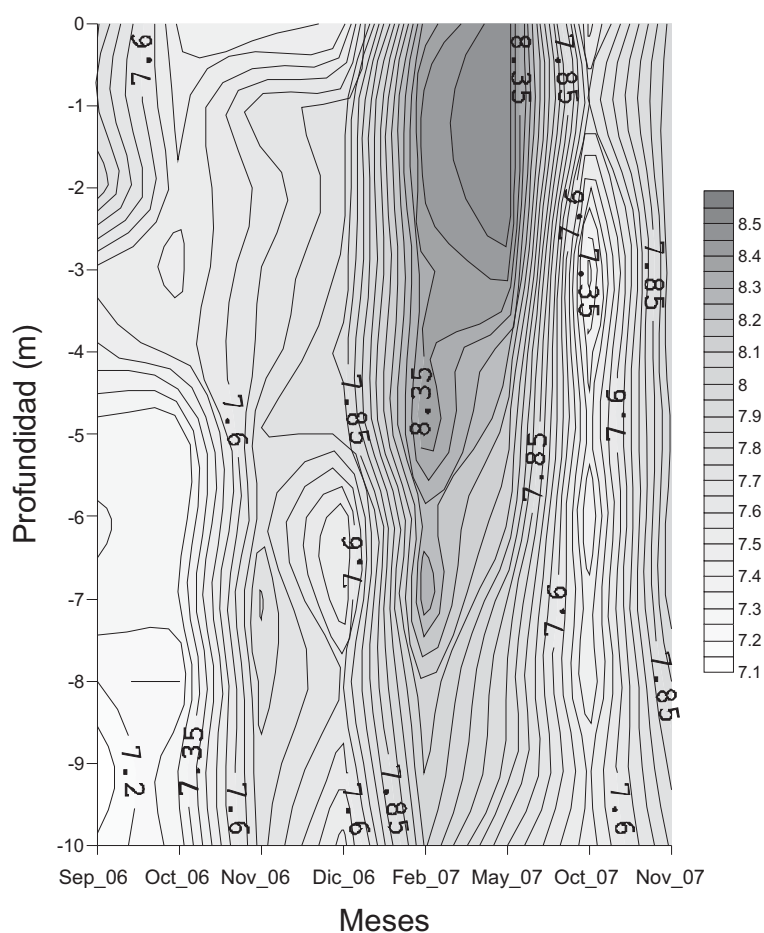

Figura 7. Variabilidad temporal y vertical del pH. pH temporal and vertical variability. 
Observaciones superiores a 8 ( $\mathrm{pH}$ alcalino) se registraron en Mayo en toda la columna de agua y en Febrero hasta los $6 \mathrm{~m}$ de profundidad, el resto de las mediciones estuvo por debajo de 8 (ligeramente alcalino).

Los registros más bajos se observaron en la cercanía del fondo del embalse, fundamentalmente durante la estratificación térmica y durante el mes de Octubre de 2007, mientras que los más altos por niveles de profundidad se detectaron generalmente a $1 \mathrm{~m}$.

En Diciembre y Octubre de 2007 se observó un comportamiento anómalo en la distribución del pH en la columna de agua, detectándose aumento de valores en profundidades intermedias seguidos por una disminución gradual.

En la comparación de medias de Febrero y Mayo, y en toda la columna de agua, se observaron diferencias significativas con el resto de los meses estudiados $(p<0.028)$, excepto para las mediciones en el fondo del embalse $(10 \mathrm{~m})$ durante el mes Mayo, donde se pierden esas diferencias.

\section{Correlaciones}

Las correlaciones más fuertes encontradas durante la estratificación térmica del embalse fueron entre profundidad y oxígeno $(r=-0.819)$, profundidad y tempeartura $(r=0.700), \mathrm{pH}$ y oxígeno $(r=0.817)$ y Eh y pxígeno $(r=0.668)$ (Tabla 4). Por el contrario durante el proceso de mezcla las correlaciones mayores resultaron entre el Eh y oxígeno $(r=0.676)$ y $\mathrm{pH}$ y oxígeno $(r=0.613)$ (Tabla 5). Finalmente, en el análisis conjunto de todos las correlaciones más elevadas fueron entre el $\mathrm{pH}$ y el oxígeno $(r=0.722) \mathrm{y}$ entre Eh y oxígeno $(r=0.678)$.

Tabla 4. Matriz de correlación de Pearson (estratificación térmica) $n=45$ y $* p<0.01$. Pearson correlation matrix (thermal stratification) $\mathrm{n}=45$ and $* \mathrm{p}<0.01$.

\begin{tabular}{lccccr}
\hline & Profundidad & OD & Eh & $\mathrm{pH}$ & $\mathrm{T}$ \\
\hline Profundidad & 1 & & & & \\
OD & $-0.819^{*}$ & 1 & & & \\
Eh & $-0.453^{*}$ & $0.668^{*}$ & 1 & & \\
$\mathrm{pH}$ & $-0.456^{*}$ & $0.817^{*}$ & $0.506^{*}$ & 1 & \\
$\mathrm{~T}$ & $-0.700^{*}$ & $0.543^{*}$ & 0.234 & 0.065 & 1 \\
\hline
\end{tabular}

Tabla 5. Matriz de correlación de Pearson (mezcla) $n=55$ y $* p<0.01$. Pearson correlation matrix (mixing) $\mathrm{n}=55$ and $*$ $\mathrm{p}<0.01$.

\begin{tabular}{lccccc}
\hline & Profundidad & OD & $\mathrm{pE}$ & $\mathrm{pH}$ & $\mathrm{Tem}$ \\
\hline Profundidad & 1 & & & & \\
OD & $-0.461^{*}$ & 1 & & & \\
Eh & -0.023 & $0.676^{*}$ & 1 & & \\
$\mathrm{pH}$ & -0.111 & $0.613^{*}$ & 0.245 & 1 & \\
$\mathrm{~T}$ & $-0.467^{*}$ & -0.200 & $-0.626^{*}$ & -0.181 & 1 \\
\hline
\end{tabular}

\section{DISCUSIÓN}

\section{Temperatura}

Según los muestreos realizados durante el 2006, el embalse resultó monomíctico, con un solo proceso de mezcla en ese año, comportamientos similares han sido reportados en otros embalses de diferentes regiones del mundo (Salmaso et al., 2003; Varekamp, 2003; González et al., 2004).

El comportamiento anómalo observado en Octubre de 2007 podría ser consecuencia de las abundantes lluvias antes del muestreo. Según información de la gerencia de Recursos Hidráulicos en Cienfuegos, el escurrimiento incorporado al embalse durante la semana anterior al muestreo fue de $3.941 \mathrm{hm}^{3}$, valor que representa casi la mitad del volumen de almacenamiento del embalse.

La entrada de esta cantidad de agua en tan poco tiempo durante el mes de Octubre del 2007, unido a la morfometría del embalse y de su cuenca de alimentación (Tabla 1), pudo haber afectado su dinámica y haber producido la mezcla que se observó entre los 2 y $9 \mathrm{~m}$ de profundidad. En esta ocasión el embalse se comportó como polimíctico, comportamiento que pudiera repetirse en períodos de intensas precipitaciones.

Los gradientes en la superficie y fondo observados en este mes pudieran relacionarse con el establecimiento de las condiciones normales correspondientes al verano en Octubre, y a la estabilización del escurrimiento del río.

\section{Oxígeno Disuelto}

Los valores más altos de saturación observados a $1 \mathrm{~m}$ de profundidad se podrían relacionar con 
los resultados de un estudio realizado en el embalse durante los años 2005 y 2006 (Comas et al., 2007), donde se encontró la mayor concentración de fitoplancton (productor de oxígeno por la actividad fotosintética) entre 0.5 y $1.5 \mathrm{~m}$.

Cuando se comparan los patrones de distribución del oxígeno los de la temperatura, se evidencia que este embalse posee una distribución termal del tipo clinógrada. Este tipo de curva se caracteriza por una concentración relativamente mayor de oxígeno cerca de la superficie, donde se desarrolla la actividad fotosintética, con una fuerte estratificación, mientras que el hipolimnion presenta concentraciones bajas de oxígeno, pudiendo llegarse a la anoxia.

Este patrón de distribución vertical de oxígeno se observa en cuerpos de agua estratificados, con una alta productividad y una reducción significativa de oxígeno en el hipolimnion, como resultado de la descomposición aeróbica de la materia orgánica generada por lo fototrofos, (Fuentes y Massol-Dayá, 2002; Sawyer et al., 2003) y puede interpretarse como un síntoma de eutrofia (Margalef, 1983).

Como es característico en las curvas clinógradas (Wetzel, 1975), en este trabajo también se evidencia que los gradientes de concentraciones de oxígeno entre la superficie y el fondo durante los períodos de circulación son mayores que los de temperatura.

Tanto la distribución de oxígeno de tipo clinógrada, como la clasificación del agua contaminada en el fondo del embalse y durante los meses de Octubre por los bajos porcentajes de saturación de oxígeno, podrían ser una respuesta al aporte de contaminantes realizado por la cuenca (residuales porcinos, vacunos y algunos asentamientos humanos).

\section{Potencial Redox}

La homogeneidad observada en el Eh en Octubre de 2007, se explica por el efecto de la mezcla del embalse producto de las lluvias intensas ocurridas la semana anterior al muestreo.

Es probable que antes de la ocurrencia de estas lluvias el patrón de distribución de Eh fuera similar al registrado durante el mes de Octubre de 2006 marcado por gradientes de poten- ciales entre el epilimnion e hipolimnion y que al ocurrir la mezcla antes mencionada provocara los valores significativamente más bajos en los primeros $6 \mathrm{~m}$ de profundidad.

\section{pH}

Los valores altos de $\mathrm{pH}$ en los meses de Febrero y Mayo podrían corresponderse con la mayor abundancia del de fitoplancton, registrada para este período por Comas et al. (2007) en este embalse, considerando que la utilización fotosintética de $\mathrm{CO}_{2}$ provoca un aumento en los valores de pH (Conde y Gorga, 1999).

Comas et al. (2007), encontraron las concentraciones más altas de microalgas entre 0.5 y $1 \mathrm{~m}$ de profundidad, lo cual también explica los valores más altos de $\mathrm{pH}$ en el metro de profundidad.

En el fondo del embalse durante los meses de estratificación se produce un reciclado de los nutrientes, que en el caso del fósforo es favorecido por los bajos valores del pH y del Eh (Moore \& Reddy, 1994). Se ha planteado que la mezcla de agua en un embalse produce una subida de nutrientes desde el fondo hacia las capas superficiales, con el consiguiente aumento de biomasa de fitoplancton (Armengol y Dolz, 2004) y de la producción primaria.

La anomalía registrada en la distribución del oxígeno disuelto, Eh y pH correspondiente a Diciembre y Octubre de 2007, consistente en un aumento en los valores de estos indicadores en profundidades intermedias, pudiera estar relacionada con procesos sucesivos de estratificación y mezcla condicionados por factores meteorológicos. Probablemente las capas de aguas profundas con valores más bajos de $\mathrm{pH}$, Eh y oxígeno se trasladaron hasta las profundidades intermedias y a su vez las capas de aguas superficiales con cifras superiores de $\mathrm{pH}$, Eh y OD ocuparon las capas más profundas.

Muchos de los cambios en la calidad del agua que no encontraban explicación cuando se analizaban en el contexto del ciclo anual, se pueden entender muy bien cuando se analizan las condiciones meteorológicas de unos pocos días antes de que se produzca los cambios detectados (Spigel \& Imberger 1987). 


\section{Correlaciones}

La fuerte correlación encontrada entre el concentración de oxígeno y la profundidad, puede ser interpretada como una manifestación del deterioro de la calidad del agua respecto a este indicador, durante la estratificación térmica del embalse.

El oxígeno estuvo además muy correlacionado con el $\mathrm{pH}$ de forma positiva. La fotosíntesis en el epilimnion incrementa la concentración de oxígeno y disminuye la concentración del $\mathrm{CO}_{2}$, produciéndose un aumento del $\mathrm{pH}$ (Wetzel, 1975), por otra parte la degradación de la materia orgánica presente en los sedimentos produce un consumo de $\mathrm{OD}$ y un aumento de $\mathrm{pH}$ por la respectiva liberación de $\mathrm{CO}_{2}$ (Bédard \& Knowles, 1991, González et al., 2004).

\section{CONCLUSIONES}

Según el patrón de evolución temporal de la temperatura, el embalse puede clasificarse como polimíctico durante el año 2007 debido a condiciones climáticas puntuales (lluvias intensas) y monomíctico en el 2006, con un patrón de distribución del oxígeno de tipo clinógrado y anómalo para el verano dada las condiciones climáticas puntuales (intensas lluvias), con $\mathrm{pH}$ ligeramente alcalino y alcalino en toda la columna de agua, mientras que el Eh se mueve en un intervalo que favorece las reacciones redox durante el período de estratificación térmica.

El proceso de estratificación térmica comenzó en Julio extendiéndose durante todo el verano, para dar comienzo a la mezcla con la llegada del invierno en Noviembre de 2006.

Los valores más bajos de todos los indicadores en estudios se encontraron en las profundidades cercanas al fondo del embalse, y las concentraciones de oxígeno registraron los valores más altos a $1 \mathrm{~m}$ de profundidad y durante los períodos de mezcla, coincidiendo con las temperaturas más bajas del año. Durante la estratificación térmica la calidad del agua puede considerarse como "dudosa" o "contaminada" según los valores de saturación de oxígeno que se midieron.

Se registró coincidencia de la termoclina y oxiclina desde el metro de profundidad hasta las capas intermedias (6 m), mientras que la redoxclina se ubicó generalmente próxima al fondo, exceptuando al final de la estratificación térmica (Octubre de 2006), que hubo coincidencia de termoclina, oxiclina y redoxclina entre los 4 y $6 \mathrm{~m}$ de profundidad.

Las mayores correlaciones encontradas fueron entre Eh y oxígeno, $\mathrm{pH}$ y temperatura así como entra $\mathrm{pH}$ y oxígeno. Finalmente, se encontró un modelo empírico que relaciona el Eh con la concentración de oxígeno y la temperatura.

El comportamiento anómalo registrado en la columna de agua en el mes de Diciembre y durante la ocurrencia de lluvias intensas, merece atención y será objeto de estudios posteriores.

\section{BIBLIOGRAFÍA}

AHLGREN, I., F. SÖRENSSON, T. WAARA \& K. VREDE. 1994. Nitrogen budgets in relation to microbial transformations in lakes. Ambio., 23(6): 367-377.

ARMENGOL, J. \& J. C. GARCÍA. 1997. Ecología de los embalses españoles. Ecosistemas, 21: 36-41.

ARMENGOL, J. \& J. DOLZ. 2004. La gestión a corto plazo del agua de los embalses. En: Ponencias del II Congreso Internacional de Ingeniería Civil, Territorio y Medio Ambiente. Santiago de Compostela. 22-24 septiembre de 2004. Ed. Colegio de Ingenieros de Caminos, Canales y Puertos. Madrid: 875-889.

AMINOT, A. \& F. ANDRIEUX. 1996. Concept and determination of exchangeable phosphate in aquatic sediments. Water Res., 30(11): 2805-2811.

APHA-AWWA-WPCF. 1992. Standard methods for the examination of water and wastewater. $18^{\text {th }}$ ed. Washington, DC. 2648 pp.

APPAN, A. \& D. S. TING. 1996. A laboratory study of sediment phosphorus flux in two tropical reservoirs. Water Sci.Technol., 34: 7-8.

BACHMANM, T. M., K. FRIESE, \& D. W. ZACHMANN. 2001. Redox and $\mathrm{pH}$ conditions in the water column and in the sediments of an acidic mining lake. Journal of Geochemical Exploration, 73: 75-86.

BÉDARD, C. \& R. KNOWLES. 1991. Hypolimnetic $\mathrm{O}_{2}$ consumption, denitrification, and methanogenesis in a thermally strati-fied lake. Can. J. Fish. 
Aquat. Sci., 48: 1048-1054.

BETANCOURT, C. \& L. TOLEDO. 2007. Descripción de los problemas ambientales del Embalse Paso Bonito y de la cuenca de alimentación. Informe Parcial de Proyecto, Programa Territorial "Conservación y Protección del Medio Ambiente", CEAC, CITMA, Cuba, 50 pp.

BEUTEL, M. W. 2006. Inhibition of ammonia release from anoxic profundal sediments in lakes using hypolimnetic oxygenation. Ecological Eng., 28: 271-279.

BOSTRÖM, B., J. A. ANDERSEN, S. FLEISCHER \& M. JANSSON. 1988. Exchange of phosphorus across the sediment-water interface. Hydrobiologia, 179: 229-244.

COMAS, A., A. MOREIRA \& S. URIZA. 2007. Cianoprocariotas (Cianobacterias, Cianoficeas) y microalgas como indicadoras de la calidad del agua en el embalse Paso Bonito, Cienfuegos, Cuba. Informe Científico Técnico. Proyecto Ramal Agencia de Medio Ambiente, CITMA, 39 pp.

CONDE, D. \& J. GORGA. 1999. Composición iónica. In: Métodos en ecología de aguas continentales. R. Arozena y D. Conde, (eds.): 65-76. D. I. R.A.C. Fac. Ciencias. Universidad de Montevideo. FUENTES, F. \& A. MASSOL-DEYÁ. 2002. Manual de laboratorio. Ecología de Microorganismos. Universidad de Puerto Rico. 202-217.

GARCÍA-GIL, L. J. \& A. CAMACHO. 2001. Anaerolimnología: pequeña guía para el muestreo en aguas microaeróbicas y anóxicas en lagos y embalses estratificados. Limnetica, 20(1): 179-186.

GEIDER, R. J. \& B. A. OSBORN. 1992. Algal photosynthesis. The Measurement of Algal Gas Exchange. Chapman \& Hall. London. 256 pp.

GONZÁLEZ, E. J., V. CARRILLO \& C. PEÑAHERRERA. 2004. Características físicas y químicas del embalse Agua Fría (parque nacional Macarao, estado Miranda, Venezuela). Acta Científica Venezolana, 55: 225-236.

HARPER, D. 1992. Eutrophication of freshwaters. Principles, problems and restoration. Chapman \& Hall. London. 327 pp.

HARRIS, G. P. 1999. Comparison of the biogeochemistry of lakes and estuaries: ecosystem processes, functional groups, hysteresis effects and interactions between macro- and microbiology. J. Marine Freshwater Res., 50: 791-811.

KASSIM, M. A. 1997. Preliminary studies on effectiveness of artificial aeration in reducing iron and manganese levels in a tropical reservoir. Proc.,
IAWQ-IWSA Joint Specialist Conf. on an Integrated System of Reservoir Management and Water Supply, Prague, Czech Republic, 123-130.

KILHAM, P. \& S. S. KILHAM. 1990. Endless summer: Internal loading processes dominate nutrient cycling in tropical lakes. Freshwat. Biol., 23: 379389.

MARGALEF, R. 1983. Limnología. Editorial Omega, Barcelona. 1010 pp.

MCGINNIS, D. F. \& J. C. LITTLE. 2002. Predicting diffused-bubble oxygen transfer rate using the discrete-bubble model. Water Res., 36: 4627-4635.

MOORE, P. A. \& K. R. REDDY. 1994. Role of Eh and $\mathrm{pH}$ on phosphorus geochemistry in sediments of Lake Okeechobee, Florida. J. Environ. Qual., 23: 955-964.

SALMASO, N., R. MOSELLO, L. GARIBALDI, F. DECET, M.C. BRIZZIO, \& P. CORDELLA. 2003. Vertical mixing as a determinant of trophic status in deep lakes: a case study from two lakes south of the Alps (Lake Garda and Lake Iseo). J. Limnol., 62 (Suppl. 1): 33-41.

SAWYER, C. N., P. L. MCCARTY, \& G. F. PARKIN. 2003. Chemistry for Environmental Engineering and Science, $5^{\text {th }}$ ed., McGraw Hill, Burr Ridge, IL 752 pp.

SPIGEL, R. H. \& J. IMBERGER. 1987. Mixing processes relevant to phytoplankton dynamics in lakes. New Zealand J. Marine Freshw. Res., 21: 361377.

TILMAN, D., S. S. KILHAM, \& P. KILHAM. 1982. Phytoplankton community ecology: The role of limiting nutrients. Ann. Rev. Ecol. Syst., 13: 349-372.

VAREKAMP, J. C. 2003. Lake contamination models for evolution towards steady state. J. Limnol., 62 (Suppl.1): 67-72.

WAGMAN, R. D., W. H. EVANS, V. B. PARKER, R. H. SCHUMM, T. HALOW, S. N. HAILEY, K. L. CHURNEY, \& R. L. NUTTAL. 1982. The NBS tables of chemical thermodynamic properties: selected values for inorganic and $\mathrm{C}_{1}$ and $\mathrm{C}_{2}$ organic substances in SI units. J. Phys. Chem., (Suppl. No 2): 392 .

WALLACE, R. D. 2007. Dynamic interaction of iron chemistry in Mantua reservoir and ferric staining in the secondary water system of Brigham City, Utah. Thesis Master of Science. Brigham Young University. $70 \mathrm{pp}$.

WETZEL, R. G. 1975. Limnology, W. B. Saunders Company, United States of America. 743 pp. 